\title{
La consulta previa, libre e informada en el Ecuador y la necesidad de su
} codificación

Free, prior and informed consultation in Ecuador and the need for its codification

David Sebastián Vázquez-Martínez david.vazquez@ucacue.edu.ec

Universidad Católica de Cuenca, Cuenca

Ecuador

https://orcid.org/0000-0002-7430-0351

Juan Carlos Erazo-Álvarez

jcerazo@ucacue.edu.ec

Universidad Católica de Cuenca, Cuenca

Ecuador

https://orcid.org/0000-0001-6480-2270

Cecilia Ivonne Narváez-Zurita

inarvaez@ucacue.edu.ec

Universidad Católica de Cuenca, Cuenca

Ecuador

https://orcid.org/0000-0002-7437-9880

Enrique Eugenio Pozo-Cabrera epozo@ucacue.edu.ec

Universidad Católica de Cuenca, Cuenca

Ecuador

https://orcid.org/0000-0003-4980-6403

Recibido: 12 de abril de 2020

Revisado: 21 de abril de 2020

Aprobado: 23 de mayo de 2020

Publicado: 08 de junio de 2020 


\title{
RESUMEN
}

La garantía de los derechos colectivos de participación de las comunas, comunidades, pueblos y nacionalidades, es uno de los retos que el Estado ecuatoriano no ha podido cumplir. El objetivo es determinar los estándares internacionales de la consulta previa libre e informada y mediante un ejercicio de control de convencionalidad determinar el grado de aplicación de los mismos en la normativa infra constitucional ecuatoriana. La investigación fue de tipo mixta con énfasis en lo cualitativo, se aplicaron métodos como el inductivo-deductivo, histórico lógico, analítico sintético y derecho comparado. Se estableció como resultado que el estado ecuatoriano no ha desarrollado normativa infra constitucional incumpliendo los estándares internacionales para la consulta como el ser previa, libre e informada demostrado en el análisis del caso Río Blanco. Como aporte se estableció la necesidad imperiosa de generar un código orgánico de consulta previa libre e informada que garantice este derecho en el Ecuador.

Descriptores: Derecho constitucional; derechos humanos colectivos; derecho a la autodeterminación; derecho comparado. (Palabras tomadas del Tesauro UNESCO).

\begin{abstract}
The guarantee of the collective participation rights of the communes, communities, towns, and nationalities is one of the challenges that the Ecuadorian State has not been able to meet. The objective is to determine the international criteria for free and informed prior consultation and, by means of a conventionality control exercise, determine the degree of application of the same in the Ecuadorian infra-constitutional regulations. The research was of a mixed type with an emphasis on the qualitative, methods such as inductivedeductive, logical historical, synthetic analytical and comparative law were applied. It was considered as a result that the Ecuadorian state has not developed infra-constitutional norms, in breach of the international problems for consultation, such as being prior, free and informed determined in the analysis of the Río Blanco case. As a contribution is the urgent need to generate an organic code of free prior consultation and information that guarantees this right in Ecuador.
\end{abstract}

Descriptors: Constitutional right; collective human rights; right to self-determination; comparative law. (Words taken from the UNESCO Thesaurus). 
David Sebastián Vázquez-Martínez; Juan Carlos Erazo-Álvarez; Cecilia Ivonne Narváez-Zurita; Enrique Eugenio Pozo-Cabrera

\section{INTRODUCCIÓN}

El Estado ecuatoriano en sintonía a la realidad internacional y local, respecto al restablecimiento de derechos de carácter colectivo de las comunidades, pueblos y nacionalidades indígenas, pueblos afroecuatorianos y montubios por medio del desarrollo de la llamada consulta previa, libre e informada (CPLI); ha adecuado dicho derecho a su normativa constitucional, por medio de un ejercicio de convencionalidad de la jurisprudencia que arrojan los fallos de la Corte Interamericana de Derechos Humanos $(\mathrm{CIDH})$ y de tratados y convenios internacionales suscritos por el Ecuador como el Convenio 169 de la Organización Internacional del Trabajo (169 de la OIT).

Desde su Constitución Política de 1998 el Ecuador adopta las iniciativas normativas internacionales desarrolladas en la materia, tutelando la consulta cuando se de actividades para la extracción de recursos naturales no renovables y evolucionando en su Constitución de la República del Ecuador 2008 (CRE 2008) con la adición de consultas pre legislativas, destinadas a la realización de consultas a comunidades, pueblos y nacionalidades indígenas previo a la adopción de leyes que puedan involucrar vulneración de sus derechos; además de incluir como beneficiarios a los pueblos afroecuatorianos y montubios.

La complicada realidad ecuatoriana respecto a la histórica adopción de políticas públicas y leyes basadas en el capitalismo desenfrenado sin bases en la sustentabilidad, en donde ha prevalecido intereses económicos por sobre derechos sociales de tercera generación y vulnerando derechos de la naturaleza; ha decantado a que por medio de luchas sociales de los pueblos y nacionalidades indígenas estos levanten fuertemente su voz que ha permanecido soslayada en el tiempo.

Este trabajo propende generar en el lector conocimientos básicos sobre la distinción de los diferentes tipos de consultas, desde su objeto y aplicación; además de demostrar la importancia de este derecho colectivo, visualizar en el lector la necesidad del desarrollo urgente de una normativa infra constitucional misma que debe desarrollar la aplicación de los estándares internacionales desarrollados en el caso Sarayaku vs Ecuador ante la CIDH que serán de aplicación directa por medio del control de convencionalidad. 
En un primer capítulo ser realizó una aproximación del concepto y tipos de la CPLI, además del control de convencionalidad y su importancia en la adecuación de las normas constitucionales e infra constitucionales tanto de las resoluciones de la CIDH como de la aplicación directa e inmediata de los tratados y convenios internacionales; en un segundo capítulo se desarrolló un análisis de la problemática de la normativa ecuatoriana al no haber desarrollado una codificación que adecúe sus estamentos a los estándares que ha expresado el derecho internacional para la aplicación de la CPLI, tomando como ejemplo el caso Río Blanco.

Como último capítulo mediante derecho comparado se hace una revisión de la experiencia de países latinoamericanos en el desarrollo de normativa interna en búsqueda de garantizar e derecho a la consulta; por último se plantea la necesidad urgente de la generación de una codificación infra constitucional que adapte la normativa ecuatoriana a la realidad contemporánea de la adecuada aplicación de la CPLI; establecer la problemática actual y avizorar los grabes conflictos socio-estatales que se generarían en el futuro si no se respeta y garantiza desde el Estado este importante derecho colectivo.

Identificada la CPLI en el contexto jurídico ecuatoriano y su falta de normativa infra constitucional, es importante preguntarse ¿Cómo mejorar los parámetros nacionales en relación a los estándares internacionales de la CPLI en el Ecuador? Bajo ese contexto, el objetivo de este artículo es generar la urgente necesidad de creación del código orgánico de aplicación de la CPLI en el Ecuador en apego a los estándares internacionales.

\section{DESARROLLO}

\section{La Consulta Previa Libre e Informada, parámetros y contexto jurídico ecuatoriano}

Marcado como un tema controversial en la palestra internacional, la CPLI ha generado gran debate tanto en su ejecución, aceptación estatal y alcance principalmente por los variados intereses en juego, por un lado, las empresas extractivistas y transnacionales que ponen sobre la mesa sus beneficios sobre las riquezas de los recursos no renovables 
(Vargas, 2018). el Estado en su papel de consultante, con la mira puesta en los réditos de las actividades extractivistas e intereses políticos en la implementación de leyes; por último, las Comunidades Pueblos y Nacionalidades Indígenas, pueblos afroecuatorianos y montubios, en su papel de consultados en búsqueda de hacer valer sus derechos colectivos (Cañarte-Sisalima, Erazo-Álvarez, Vázquez-Calle, \& Narváez-Zurita, 2020). Motivo de aclaración dentro del presente estudio será el marcar las diferencias entre los tipos de consultas; además de sustanciar la importancia en el cumplimiento de estándares internacionales mediante el análisis del llamado control de convencionalidad, las experiencias en la generación de normativas infra constitucionales de países hermanos y la aplicación de los mismos en el caso Rio Blanco en el Ecuador.

La CPLI entendida como un derecho colectivo se respalda internacionalmente por medio del Convenio 169 de la Organización Internacional del Trabajo y la Declaración de las Naciones Unidas sobre los Derechos de los Pueblos Indígenas; constitucionalmente, teniendo como beneficiarios a las comunidades pueblos y nacionalidades indígenas a través de su Carta Magna en los numerales 7 y 17 del artículo 57 y garantizando los mismos derechos colectivos para los pueblos afroecuatorianos y montubios en el artículo 58 y 59 respectivamente de la norma ibidem ; infra constitucionalmente, para la consulta pre legislativa por medio del Instructivo de Aplicación de Consulta Prelegislativa de la Asamblea Nacional; jurisprudencia, por medio de la atribución de la Corte Constitucional para expedir sentencias que constituyan jurisprudencia vinculante prevista en el artículo 436 numeral 6 de la Constitución de la República, tenemos la Sentencia 001-10-SIN-CC referente a la Ley de Minería (precedente respecto a la diferenciación de consultas) y la sentencia dictada por la CIDH en el caso del Pueblo Sarayacu vs Ecuador (determinación de parámetros de aplicación del a CPLI).

En el contexto internacional, en su artículo 19 la Declaración de las Naciones Unidas sobre los Derechos de los Pueblos Indígenas del 2007 señala: que los Estados de buena fe cooperarán y celebrarán consultas con los pueblos indígenas interesados por intermedio de sus instituciones representativas antes de la adopción y aplicación de 
medidas administrativas o legislativas que los perturben, con la finalidad de alcanzar su consentimiento informado, libre y previo (ONU, 2007).

En el año 1989 la Organización Internacional del Trabajo (OIT) acoge el Convenio 169 sobre Pueblos Indígenas y tribales adoptado en la Organización Internacional del Trabajo (OIT), ratificado por Ecuador en 1998 y Publicado en el Registro Oficial 206 de 07 de junio de 1999; en los numerales 1 y 2 del artículo 6, se establece que los gobiernos estarán obligados a consultar a los pueblos que muestren interés, por medio de procedimientos adecuados y, a través de las instituciones que los represente, siempre que se promuevan medidas administrativas o legislativas capaces de perturbar de manera directa; dichas consultas deberán efectuarse de una manera apropiada a las circunstancias y de buena fe, con el fin de celebrar un consentimiento en base de un acuerdo sobre los temas a tratar. (OIT, 1989).

Los derechos otorgados a las comunidades, pueblos y nacionalidades indígenas, montubias y afroecuatorianas establecidos en la Carta Magna Ecuatoriana en su artículo 56; históricamente determinados como colectivos y de tercera generación por sus características a ser susceptibles de individualizar a los beneficiarios, en contra posición a los derechos difusos (Grijalva, 2009). Este aporte nos brinda una herramienta fundamental a favor de la distinción entre los tipos de consultas, entendiendo de esta manera que la CPLI del artículo 57 numeral 7 y la CPL del artículo 57 numeral 17 que están revestidos por la protección de derechos colectivos dado que se puede individualizar a sus beneficiarios, en tanto que la Consulta Ambiental garantizada en el artículo 398 es aplicable a toda una comunidad, es decir no está particularizado un grupo o comunidad especialmente favorecido teniendo una clara característica de difuso (López, 2016).

A continuación, se detalla las implicaciones de los derechos colectivos relacionados a los dos tipos de consultas que se garantizan en el Ecuador a las comunas, comunidades, pueblos y nacionalidades indígenas, pueblos afroecuatorianos y montubios:

Art. 57.- Se reconoce y garantizará a las comunas, comunidades, pueblos y nacionalidades indígenas, de conformidad con la Constitución y con los pactos, 
convenios, declaraciones y demás instrumentos internacionales de derechos humanos, los siguientes derechos colectivos

En su numeral 7 establece que "La consulta previa, libre e informada, dentro de un plazo razonable, sobre planes y programas de prospección, explotación y comercialización de recursos no renovables que se encuentren en sus tierras y que puedan afectarles ambiental o culturalmente; participar en los beneficios que esos proyectos reporten y recibir indemnizaciones por los perjuicios sociales, culturales y ambientales que les causen. La consulta que deban realizar las autoridades competentes será obligatoria y oportuna.

Si no se obtuviese el consentimiento de la comunidad consultada, se procederá conforme a la Constitución y la ley". (Asamblea Nacional Constituyente, 2008).

Este primer tipo de consulta conocida propiamente dicha como Consulta Previa Libre e Informada, hace referencia a la intención del legislador de garantizar por medio de este derecho colectivo de la obligatoriedad por parte del Estado de consultar a comunas, comunidades, pueblos y nacionalidades indígenas y que su decisión sea tomada en cuenta aunque no de manera vinculante sobre planes y programas de prospección, explotación y comercialización de recursos no renovables que se encuentren en sus tierras y que puedan afectarles ambiental o culturalmente; además de ser beneficiados de los réditos que puedan generar dichas explotaciones en sus tierras. Vulneraciones a tal derecho en especial ante la falta de una normativa infra constitucional ha conllevado a demandas en el ámbito local como es el caso de la acción de protección del caso Rio Blanco e internacional como el presentado ante la CIDH en el caso del Pueblo Sarayacu vs Ecuador.

Otro tipo de consulta colectiva es la contemplada en su numeral 17 de la norma ibidem, donde se establece que estas comunidades pueblos y nacionalidades gozarán del derecho a ser consultados previo de la adopción de una disposición legislativa que perturbe cualquiera de sus derechos catalogados como colectivos (Asamblea Nacional Constituyente, 2008). Este tipo de consulta dirigida a comunas, comunidades, pueblos y nacionalidades indígenas, pueblos afroecuatorianos y montubios; respalda la 
obligatoriedad del Estado de consultar a este colectivo sobre la promulgación de futuras leyes que puedan ser lesivas a sus derechos, so pena a que los afectados estarán en sus facultades de reclamar tanto judicial como constitucionalmente, tal como ocurrió en la acción de protección que conllevó la Sentencia 001-10-SIN-CC dictada por el Pleno de la Corte Constitucional del Ecuador, referente a los casos 0008-09-IN y 0011-09-IN sobre la Ley de Minería; misma que entre otros, marcó los preceptos y características de distinción entre los tipos de consultas que se han realizado en Ecuador (Machuca, 2014). Cabe recalcar que el artículo citado en su inciso final establece que de manera expresa el Estado ecuatoriano está en la obligación de cumplir los estándares internacionales relacionados a estos tipos de consultas por medio del llamado control de convencionalidad al citar que se harán cumplir estos derechos conforme los pactos, convenios, declaraciones y demás instrumentos internacionales de derechos humanos.

El derecho a la Consulta Ambiental, de características claramente difuso y establecido en el artículo 98 de la carta magna, nace de la necesidad de poder garantizar a la comunidad en general sobre cualquier decisión o autorización estatal que ponga en riesgo al ambiente; un punto en discusión y que genera controversia es en el caso de existir oposición mayoritaria será el mismo Estado quien mediante resolución motivada tendría la última palabra en la decisión, no siendo vinculante la voluntad de la comunidad (Asamblea Nacional Constituyente, 2008).

Dentro de los llamados precedentes jurisprudenciales en relación a la atribución de la Corte Constitucional para expedir sentencias que constituyan jurisprudencia vinculante; tenemos a una de las más importantes dentro de nuestro campo de análisis, la denominada Sentencia de la Ley de Minería No.001-10-SIN-CC dictada el 8 de marzo de 2010, demanda que reclama entre otros el derecho a la consulta prelegislativa, en dicha sentencia se evidencia un avance en la distinción entre los tres tipos de consultas, primero separando a la CPLI y la Consulta Ambiental de carácter colectivo y la Consulta ambiental del artículo 398 de característica difusa, entendiendo que las dos primeras tienen un beneficiario definido y la última destinada a un universo general. 
El segundo avance de la Sentencia en el campo de la Consulta prelegislativa es el establecimiento de cuatro faces para su realización, contando con fase de preparación de la consulta, fase de convocatoria, fase de información y realización y fase de análisis de resultados y cierre de la consulta; estas deben estar revestidas de doce reglas y procedimientos para su correcta aplicación, mismos que también se aplicarán a la Consulta Previa Libre e Informada (Porras \& Romero, 2012).

En revisión de la normativa desarrollada por el Ecuador tenemos al instructivo para la aplicación de la Consulta Prelegislativa y a la ley Orgánica de Participación Ciudadana; el primero, discutido por su inconstitucionalidad al no respetarse la reserva de ley y no considerar como vinculante la decisión de la comunidad consultada, establece que la Consulta pre legislativa estará a cargo de la Asamblea Nacional por medio de una comisión especializada ocasional o permanente; determina cuatro faces para la realización de la consulta, además de fijar principios elementales al ejercer este derecho como el de oportunidad, de autonomía, de buena fe, de interculturalidad y plurinacionalidad, plazo razonable, de información veraz y suficiente.

La ley Orgánica de Participación Ciudadana en cambio hace hincapié en la diferenciación entre la CPLI y la Consulta Ambiental basada nuevamente sobre a quien se consultará, además refuerza la controvertida decisión del Estado de catalogar como no vinculante la voluntad de la comunidad, dado que al haber oposición mayoritaria será el Estado quien tomará la última palabra.

\section{La inexistencia de una normativa infra constitucional y su incidencia en la consulta previa, libre e informada}

Con la finalidad evidenciar el Estado del desarrollo de normativa ecuatoriana en relación a la adopción de los estándares internacionales de la Consulta Previa Libre e Informada se realizó una revisión de la sentencia de primera y segunda instancia del caso Río Blanco, el cual es hoy por hoy un caso emblemático respecto de la protección de aquel derecho colectivo (Reyes-Cueva, Erazo-Álvarez, Borja-Pozo, \& Narváez-Zurita, 2020). 
En ese contexto el proyecto minero con 90 millones de dólares en inversión a cargo de la empresa China Ecuagoldmining (fundada en Hong Kong en 1990) ubicada en la comunidad de Río Blanco, perteneciente a la parroquia de Molleturo, en Cuenca; sus concesiones están dentro del bosque protector Molleturo-Mollepungo, colindante con el Parque Nacional Cajas, ecosistema de páramo categorizado por su gran importancia biológica como Reserva Mundial de la Biósfera. En fase de explotación desde el 11 de agosto de 2016, una vida útil de 11 años y un potencial de 4,3 millones onzas de plata y 605.000 onzas de oro; el Estado ecuatoriano ha impulsado con gran decisión al que considera uno de los proyectos iniciales que catapultará al Ecuador como un Estado minero. (Presidencia de la República del Ecuador, 2017).

Varios conflictos de carácter social se han dado en la comunidad, entre comuneros que están a favor y en contra de la explotación; los que manifiestan su inconformidad aducen graves vulneraciones de derechos constitucionales tales como el derecho a un debido proceso; a la libertad y seguridad personal (vías de acceso a la comunidad fueron privatizadas); a la salud (al haber recibido maltrato físico y psicológico por la compañía); derecho a una vivienda adecuada(personas han abandonado sus viviendas por el conflicto); derecho a la libertad sindical y derechos laborales (se les ha impedido generar un sindicato); y el derecho a la participación motivo de nuestro análisis, dado que la comunidad manifiesta que se les ha negado su acceso a una consulta sobre el proyecto de manera previa, libre e informada a las comunidades indígenas de la zona de influencia del proyecto, a lo que el Estado manifestó que la empresa "ha puesto en práctica un exhaustivo Plan de Relacionamiento Comunitario en las comunidades de Cochapamba, Río Blanco, San Pedro de Yumate y Molleturo" (Plan V, 2018).

El 11 de agosto de 2017 inician las protestas cuando comuneros acamparon a la entrada de la mina impidiendo su funcionamiento, siendo el punto más álgido de las mismas el 10 de octubre del mismo año con enfrentamientos que dejaron personas heridas y detenidos; en búsqueda de reestablecer sus derechos colectivos el jueves 17 de mayo de 2018, Félix María Gutama, Arariwa Sigcha Vele (presidente de la Foa) y Yaku Pérez (Ecuarunari y Caoi) presentan ante el Juez de primera instancia Serrano Arizaga Oswaldo 
Paúl un proceso de Garantía jurisdiccional en búsqueda de una Medida cautelar que pretende la suspensión inmediata del acto administrativo que autoriza la explotación en Rio Blanco hasta que demuestren haber cumplido con el derecho a la consulta previa libre e informada y haber alcanzado el consentimiento de la comunidades de la parroquia Molleturo del Cantón Cuenca, Provincia del Azuay, presentado en contra de la Ing. Rebeca Illescas Ministra de Minas y Lcdo. Tarsicio Granizo Ministro del Ambiente (El Comercio, 2018).

El proceso número: 01333-2018-03145, que en principio inició como una medida cautelar autónoma, para posteriormente en base al principio jura novit curia mutar a una medida cautelar conjunta con una acción de protección, en audiencia y luego de escuchar la intervención de los sujetos procesales, varios amicus curiaes y la lectura de múltiples informes como el de la Defensoría del Pueblo (negando la existencia de una CPLI en Río Blanco) el juez resolvió aceptar la acción de protección de derecho al debido proceso de la Consulta Previa, ordenando como medida de restitución al derecho vulnerado que se realice la CPLI conforme al convenio 169 de la OIT (denotando la inexistencia de normativa infra constitucional) en consecuencia ordena a todas las autoridades accionadas suspender la acción de explotación en el sector de Río Blanco, además la desmilitarización gradual y paulatina de los sectores en problema, precautelando la integridad de los miembros de la comunidad y evitando sobre todo conflictos (Acción de Protección Primera Instancia caso Río Blanco, 2018).

En denotada inconformidad a la sentencia de primera instancia, el Estado plantea una apelación ante la Sala especializada de lo Civil y Mercantil de la Corte Provincial de Justicia de Azuay, misma que el 3 de agosto de 2018 en sentencia confirma el fallo de primer nivel negando la apelación; además, invalida la medida de restitución del derecho vulnerado, por cuanto, considera que el pueblo ya fue consultado el 4 de febrero de 2018 con la pregunta 5 del referéndum llevado por el Estado ¿Está usted de acuerdo con enmendar la Constitución de la República del Ecuador para que se prohíba sin excepción la minería metálica en todas sus etapas, en áreas protegidas, zonas intangibles y centros urbanos, de conformidad con lo establecido en el Anexo 5?, donde los habitantes de la 
parroquia Molleturo manifiestan: $67.80 \%$ por el SI, y el $32.20 \%$ por el NO. (Consejo Nacional Electoral, 2018) Los accionados al haber agotado los recursos ordinarios y extraordinarios, el 14 de septiembre de 2018 presentan una acción Extraordinaria de Protección (por resolver) en contra de la sentencia de primera instancia, a la cual consideran que vulneran sus derechos constitucionales.

Al analizar la motivación del juez de primera instancia notamos que invocando el acuerdo 169 de la OIT, que determina que el Estado en su papel de consultante debe realizar la consulta de buena fe y que en el caso de Río Blanco el Ecuador falló al realizar la CPLI dado que debía haberse realizado bajo dicho parámetro; esto sin duda nos deja una gran incógnita que está ligada a la buena fe por parte del Estado, con preguntas como si ¿Se puede estandarizar la llamada buena fe?, ¿La idea de buena fe es la misma en Ecuador que en el resto de países? y sobre todo ¿Puede ser imparcial el consultante en el mecanismo de consulta, a sus propios intereses políticos o económicos?

En relación a la problemática expuesta se debe desarrollar una normativa infra constitucional que estandarice y desarrolle en la consulta las características de previa, de libre de informada; además donde ser establezca de manera clara e propósito, la procedencia, el objeto; temas transcendentales como ¿Quiénes son objeto de la consulta?, ¿Cómo se debe consultar a la comunidad o comunidades?, ¿La decisión de la comunidad es vinculante? y ¿Cuáles serán los efectos de la consulta?; en ese sentido vale analizar cada uno de estos componentes.

Teniendo en cuenta que la consulta deberá ser informada, se entiende que el acceso a dicha información por parte de la comunidad tiene que ser en un contexto sencillo, además de oportuno, en su propio lenguaje y de ser necesario puedan contar con asesoría técnica (OIT, 2009). Esto quiere decir que la consulta en ningún momento puede ser considerada una simple asamblea o comversatorio, al contrario esta deberá ser en el idioma y en contexto que la comunidad requiera tomando para esto un plazo razonable para poder entender la magnitud del proyecto a ejecutar o la ley que se desea implemnentar. 
La Consulta tiene el deber de ser permanente y previa a la adopción de medidas administrativas o normativas que puedan afectar a los sujetos a consultar; conforme consta en la constitución en los numerales 7 y 17 del artículo 57, respaldado internacionalmente en el convenio 169 de la OIT y en el artículo 19 de la Declaración de las Naciones Unidas sobre los Derechos de los Pueblos Indígenas, con el fin de que los mismos recaben toda la información necesaria tanto previo al arranque del proyecto 0 antes de la presentación del plan legislativo; de no cumplirse se estaría realizando la consulta como un mero trámite (Clavero, 2013).

Cuando se habla que uno de los requisitos para que la consulta previa tenga legitimidad es que el Estado en el papel de garantista ejecute la consulta en el marco de la libertad, entendiendo que la misma no puede ser producto de manipulación, dominación o amenaza; esto en el riesgo de que por la injerencia de intereses externos se pueda ver vulnerada la voluntad de la comunidad (Carrión, 2012).

\section{Necesidad De Una Ley Orgánica Para La Consulta Previa Libre e Informada}

Dentro del análisis realizado, está la necesidad de hacer una sucinta revisión de lo que sucede en américa Latina respecto a la aplicación de la CPLI, es decir realizar una evaluación y comparación de que es lo que sucede en distintos países en su responsabilidad de adoptar este derecho colectivo y la generación de sus normativas internas, por medio del llamado derecho comparado, que no es más que un método de análisis de varios sistemas jurídicos (Tamayo, 2005).

Dentro del análisis de la consulta previa libre e informada en latinoamérica ser tomado en cuenta algunos países en donde la proporción de comunidades pueblos y nacionalidades indígenas con respecto al resto de la población es importante, por ejemplo en Chile en dónde el $11.11 \%$ de su población es considerada como indígena este país ha ratificado el convenio 169 de la OIT en el año 2008 de la misma forma Guatemala que es uno de los países con el porcentaje de población indígena más alto llegando a un $41 \%$ y 23 pueblos indígenas ratificó en el año 1996 el convenio 169 del OIT; otro caso analizar es el de Costa Rica con ocho pueblos indígenas, el $24 \%$ de su territorio dentro de dichas 
comunidades y el 2.4\% de su población identificada como indígena ratificó en 1993 el convenio 169 de la OIT; teniendo en cuenta que en América Latina el último país en ratificar dicho convenio asido Nicaragua y el país que hasta la fecha no lo ha hecho ha sido Panamá (Organización Internacional del Trabajo, 2016).

Es menester de este estudio el identificar el manejo normativo de los Estados que han ratificado el convenio 169 de la OIT y revisión de la normativa interna desarrollada por los Estados que han ratificado el convenio de análisis tenemos un caso especial que es el colombiano y el peruano; en ese sentido el Estado colombiano que fue el primer país en ratificar el convenio 169 de la OIT y de la misma forma el primero en generar normativa infra constitucional en el año 1998 con la expedición del Decreto $N^{\circ} 1320$ Y 15 años después en el año 2013 con una Directiva Presidencial, la cual determina que la responsabilidad de la consulta en temas administrativos recae en el Ministerio Interior por medio de la dirección de consulta previa; $Y$ en tema de medidas legislativas a su vez a la dirección de Asuntos Indígenas ROM y Minorías del mismo ministerio, estos avances por parte del Estado colombiano ha demostrado la eficacia de la pronta generación de normativas internas dando como resultado alrededor de 4000 acuerdos protocolizados entre el gobierno colombiano y las comunidades pueblos y nacionalidades indígenas consultadas (Naciones Unidas Derechos Humanos Oficina del alto comisionado para los Derechos Humanos Colombia).

Caso contrario a la generación de normativa, se puede citar el de los Estados de Costa Rica y Guatemala; en el primer caso en donde a partir de Del año 2015 se generado varios debates sobre la generación de una ley que desarrolle la consulta previa sin llegar a un consenso, Guatemala ha sido otro de los países en donde las discrepancias entre el ejecutivo y los grupos indígenas ha desembocado en incipientes avances respecto a normativas a favor de la protección de estos derechos (Fundación para el Debido Proceso, 2015).

El caso de Chile que conjuntamente con Perú y Colómbia se ha interesado en impulsar el desarrollo de la consulta previa libre informada en su normativa interna es rescatable, revisando su historia en el año 2009 tiene su primer intento de reglamentar aquel derecho 
mediante el Decreto Supremo № 124 del Ministerio de Planificación qué fue de carácter transitorio, para posteriormente expedir dos regulaciones el Decreto Supremo $N^{\circ} 66$ de 2013 designado para consultas Sobre resoluciones administrativas que puedan afectar derechos a las comunidades indígenas, y un segundo el Decreto Supremo $N^{\circ} 40$ de 2012 Relacionado a la consulta ambiental; cabe realcar que estos dos decretos han sido de vital importancia para que el Estado chileno regule temas como su responsabilidad en la ejecución de la consulta por medio del ministerio de Desarrollo Social, además de otros como las etapas en la consulta, responsabilidad, procedencia, principios a respetar y conceptos de identificación de la afectación directa que ayudan a determinar quiénes son objeto de este tipo de consultas (Vásquez-Ayerve, Narváez-Zurita, Pozo-Cabrera, \& Erazo-Álvarez, 2020).

Otro país que ha realizado avances importantes en el desarrollo normativo de este derecho ha sido México, por medio del Estado de San Luis de Potosí ha desarrollado una ley de consulta; además, Perú en los últimos años ha desarrollado de manera eficiente su normativa interna con la expedición de la ley de consulta previa y su reglamento. Caso contrario a países que de manera exitosa han generado su normativa interna, tenemos a Ecuador y Bolivia; que en el caso ecuatoriano solamente ha expedido un reglamento que regula la consulta de proyectos de ley, dejando hasta la fecha Si normativa a la consulta previa libre informada sobre resoluciones administrativas (Instituto Interamericano de Derechos Humanos, 2016).

De la revisón de los casos latininoamericanos se evidencia la necesidad imperiosa de que los Estados generen sus propias normativas infraconstitucionales; de esta realidad no se escapa el Ecuador, en donde la carencia de un Código Orgánico de Consulta Previa que regule temas como la finalidad de la consulta; principios como el de oportunidad, interculturalidad, buena fe, plazo razonable, imparcialidad e información oportuna; establezca de manera clara los sujetos de consulta, identificación de las comunidades, pueblos y nacionalidades; marque de manera clara las etápas del proceso de consulta, la entidad encargada de la consulta y como se financiará la misma (Organización Internacional del Trabajo, 2016). 


\section{MÉTODO}

Se aplicó la metodología de carácter no experimental puesto que las variables no fueron manipuladas. El tipo fue mixto, es decir cuantitativa-cualitativa, con énfasis en lo cualitativo donde se recabó información heterogénea de varias fuentes de tipo documental - bibliográfica, de un carácter descriptivo que sirvió para especificar las diferentes causas que aportan a la problemática (Pesántez-Ochoa, Erazo-Álvarez, JVázquez-Calle, \& Narváez-Zurita, 2020). Mediante la aplicación de métodos como el inductivo-deductivo, que va de lo general a lo particular y viceversa. Otro método utilizado fue el Histórico-Lógico, basado en el estudio de la trayectoria de la problemática en el tiempo, de una manera cronológica (Tamayo, 2005).

\section{Universo de estudio y tratamiento muestral}

Mediante la relialización de un muestreo por conveniencia que es un procedimiento no probabilístico al azar formado por el caso accesible y la disponibilidad de personas naturales que serán parte de la investigación, que para el estudio fueron 10 docentes universitarios en el áres del Derecho Constitucional.

\section{Tratamiento estadistico de la información}

Para la obtención de los datos se creó por medio de la plataforma de formularios de Gloole (https://docs.google.com/forms/) un cuestionario con varias preguntas agrupadas por variables; para la tabulación y representación gráfica se utilizó Microsoft Exel versión 2019 (Erazo-Álvarez \& Narváez-Zurita, 2020). 


\section{RESULTADO}

A continuación se muestran la Tabla 1 y la Figura 1 con los resulados obtenidos, clasificados según las diferentes variables establecidas.

\section{Tabla 1}

Resultados de la encuesta realizada.

VARIABLE

Necesidad

de

Codificación

Consulta no vinculante

Adopción de estándares internacionales

Estandar de informada

Estandar de libre

Estandar de previa

Aplicación de la CPLI

Vulneración de derechos colectivos

\section{RESPUESTA}

$\mathrm{Si}$

No

$\mathrm{Si}$

No

$\mathrm{Si}$

No

$\mathrm{Si}$

No

$\mathrm{Si}$

No

$\mathrm{Si}$

No

$\mathrm{Si}$

No

$\mathrm{Si}$

No

\section{RESULTADO}

$100 \%$

$0 \%$

$20 \%$

$80 \%$

$30 \%$

$70 \%$

$20 \%$

$80 \%$

$30 \%$

$70 \%$

$20 \%$

$80 \%$

$20 \%$

$80 \%$

$30 \%$

$70 \%$

Fuente: Investigación de campo 


\section{Resultados}

Considera necesaria la creación de una normativa

Considera positiva que la decisión no sea vinculante

Se ha adoptado los estándares internacionales

Se cumple con el carácter de informada

Se cumple con el carácter de libre

Se cumple con el carácter de previa

Se garantiza los derechos del al CPLI de la CRE 2008

En el Ecuador se vulneran los derechos colectivos

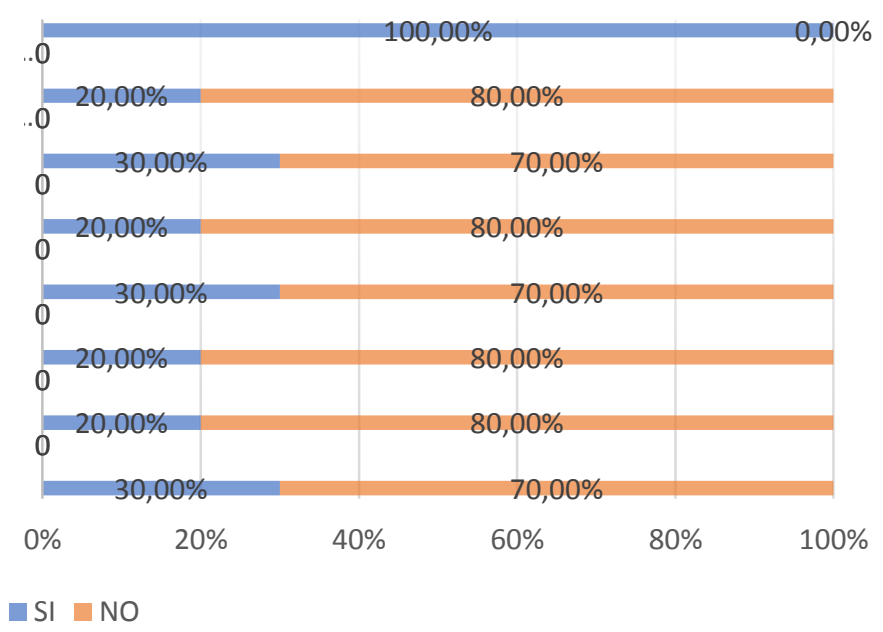

Figura 1 Representación gráfica de los resultados. Fuente: Investigación de campo

Como resultado se puede rescatar que la mayoría de los investigados consideran que existe una clara vulneración de derechos colectivos en el Ecuador y que de tales derechos es la CPLI el que menos se garantiza por parte del Estado. Respecto a los estándares la gran mayoría de los consultados determinan, que el estado no realiza la adaptación de la información de manera técnica para que la comunidad entienda las partes esenciales de la consulta y tampoco usando el idioma que maneja la comunidad; respecto al carácter de libre, consideran mayoritariamente que existe la aplicación de amenazas, coerción o presión a las comunidades, pueblos y nacionalidades por intereses económicos. En lo concerniente al requisito de que la consulta debe ser previa en su generalidad opinaron que la consulta en el Ecuador pocas veces se la realiza antes de la ejecución de planes y programas de prospección, explotación y comercialización de recursos no renovables.

Respecto al Control de Convencionalidad efectuada a la normativa ecuatoriana en relación de los estándares internacionales contemplados en el Convenio 169 de la OIT, los encuestados coinciden en que el Estado ha adoptado dichos parámetros y dentro de 
sus comentarios aducen que la razón de esto es la falta de una normativa infra constitucional que desarrolle los mismos. Respecto a la falta de consentimiento del grupo consultado, se procederá conforme a la Constitución y la ley, dando a entender que la última palabra la tendrá el consultante, pocos encuestados coinciden en que están de acuerdo en que la voluntad de los consultados no sea vinculante en la decisión final de ejecución; en contraposición a tal idea todos consideran necesaria la creación de una normativa infra constitucional (Código Orgánico) que desarrolle los estándares internacionales de la CPLI en el Ecuador.

\section{PROPUESTA}

En el devenir de la investigación se ha denotado que los estándares internacionales de la CPLI establecidos en el Convenio 169 de la OIT y la jurisprudencia vinculante de la Corte Interamericana de Derechos Humanos mediante la sentencia del caso Sarayaku vs Ecuador, no se cumplen en la realidad ecuatoriana; ¿Cuáles son las razones? Pues la principal es la falta de una normativa infraconstitcional, que deasrrolle los estándares de previa, libre e informada.

Como aporte a la investigación se a propuesto la necesidad imperiosa de la creación de un proyecto del ley de iniciativa legislativa conforme el artículo 133 numeral 1 de la Constitución ecuatoriana (Asamblea Nacional Constituyente, 2008), dicha ley tedría el carácter de Orgánica al regular el ejercicio de derechos constitucionales de acuerdo al artículo 133 numeral 2 ibídem; para garantizar que dicha norma garantice de forma adecuada el derecho colectivo de estudio es necesario que dicho proyecto parta de una adecuada evaluación del estado actual de la aplicación de la CPLI en el Ecuador, el aporte de experiencias, recabar necesidades de los consutados y el apoyo estatal, que a continuación se detallan:

1. Invitación a representantes de organismos internacionales (OIT y ONU), con el fin de realizar una evaluación del cumplimiento de estándares internacionales de la CPLI en el Ecuador. 
2. Reunión con representantes de las principales comunas, comunidades, pueblos y nacionalidades indígenas, pueblos afroecuatorianos y montubios; con la finalidad de recabar información sobre sus realidades y exigencias en relación a la CPLI.

3. Reunión con representantes de los estados de Perú, Colombia y Chile; además de actores de comunas, comunidades, pueblos y nacionalidades Indígenas de los mismos con la finalidad de recabar información sobre sus experiencias en el desarrollo y aplicación de normativa infra constitucional en sus Estados.

4. Creación de un Código Orgánico para la aplicación de la Consulta Previa Libre e Informada y de ser necesario el desarrollo de un reglamento (para el mismo se contará con la participación de actores de comunas, comunidades, pueblos y nacionalidades indígenas; además de representantes del ejecutivo y legislativo ). 


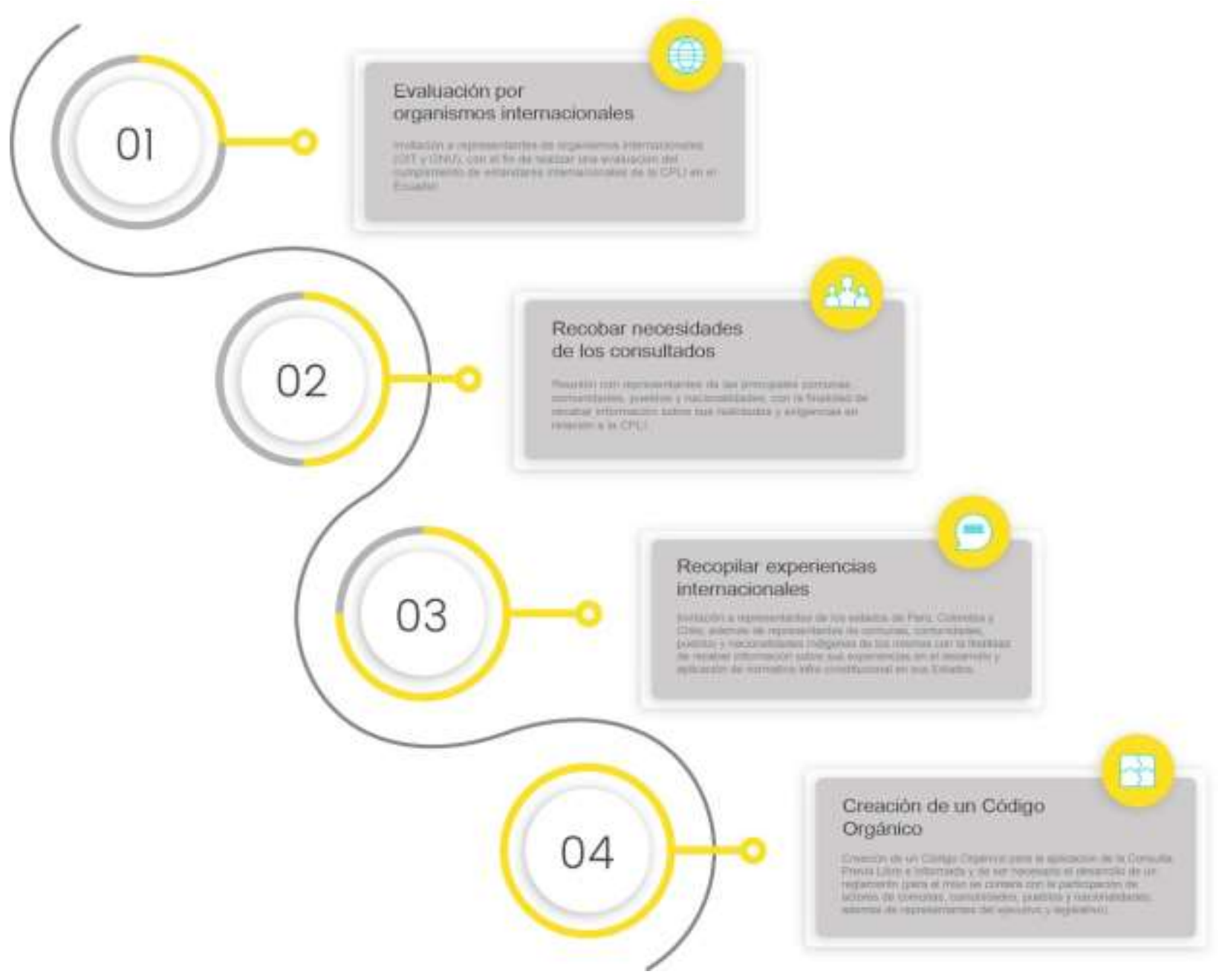

Figura 2 Representación gáfica de la propuesta. Fuente: Elaboración propia

\section{DISCUSIÓN}

El aporte internacional al desarrollo de la consulta previa, libre e informada es de suma importancia teniendo en cuenta a la Declaración de las Naciones Unidas sobre los Derechos de los Pueblos Indígenas y el convenio 169 de la Organización Mundial del Trabajo, marcando las pautas a seguir para los Estados que han ratificado los mismos; este derecho colectivo que beneficia a las comunidades, pueblos y nacionalidades en el Ecuador también ha sido garantizado mediante jurisprudencia nacional vinculante como es la Sentencia Ley de Minería No.001-10-SIN-CC que determinó la diferenciación entre 
los variados tipos de consultas por su objeto y la jurisprudencia internacional obtenida del caso Sarayaku vs Ecuador que especificó los estándares a aplicar en la CPLI.

Motivo de debate es determinar ¿Por qué razón el Ecuador no ha generado normativa que garantice este derecho constitucional que favorece la autodeterminación de la comunidades pueblos y nacionalidades, por medio del llamado control de convencionalidad?, los encuestados en este estudio han determinado que como consecuencia de esta falta de norma, se han generado conflictos entre quienes deben ser consultados (comunidades, pueblos y nacionalidades), el consultante (estado) y posibles terceros (empresas extractivistas); problemas que fueron evidenciados en el análisis del caso Río Blanco en Ecuador.

El estado tendrá sus razones para no haber generado un proyecto de ley, que desde el punto de vista personal están ligadas al peligro que siente el estado ante la posibilidad de empoderar de estos derechos de participación a un colectivo que tiene en sus tierras riquezas que son de interés directo del Estado e inversionistas (Vargas-Araujo, 2018).

Como aporte a la problemática analizada está el visibilizar el vacío y generar en el lector la necesidad imperiosa de exigir al Estado la generación de una normativa de tipo orgánica que regule temas como la finalidad de la consulta; principios como el de oportunidad, interculturalidad, buena fe, plazo razonable, imparcialidad e información oportuna; establezca de manera clara los sujetos de consulta, identificación de los pueblos y nacionalidades; marque de manera clara las etápas del proceso de consulta, la entidad encargada de la consulta y como se financiará la misma.

\section{REFERENCIAS CONSULTADAS}

Acción de Protección Primera Instancia caso Río Blanco. [First Instance Protection Action in the Río Blanco case]., 01333201803145 (Unidad Judicial Civil de Cuenca 18 de de mayo de 2018).

Angélica Porras, J. R. (2012). Guía de jurisprudencia constitucional ecuatoriana [Guide to Ecuadorian constitutional jurisprudence]. Quito: Centro de estudios y difusión del derecho Constitucional (CEDEC). 
Aparicio , W. M. (2008). Derechos:Enunciación y Principios de Aplicación. En Ramiro Ávila, Agustín Grijalva y Rubén Martínez (ed) "Derechos Constitucionales. La Constitución ecuatoriana de 2008 en perspectiva". Quito: Ministerio de Justicia y Derechos Humanos y Tribunal Constitucional.

Asamblea Nacional Constituyente. (20 de Agosto de 2008). Constitución Política del Ecuador [Political Constitution of Ecuador]. Decreto Legislativo. Montecristi, Manabí, Ecuador: Registro Oficial 449 .

Cañarte-Sisalima, C. B., Erazo-Álvarez, J. C., Vázquez-Calle, J. L., \& Narváez-Zurita, C. I. (2020). Derecho al proceso en el cometimiento de infracciones administrativas por propietarios de locales comerciales [Right to process in the commission of administrative infractions by owners of commercial premises]. Iustitia Socialis, 273294. doi:http://dx.doi.org/10.35381/racji.v5i1.612

Carrión, P. (2012). Análisis de la Consulta Previa, Libre e Informada. [Analysis of the Free, Prior and Informed Consultation.]. Quito: Centro Ecuatoriano de Derecho Ambiental, Fundación Konrad Adenauer.

Clavero, B. (2013). Consulta y Consentimiento Previo Libre e Informado a la luz del derecho Internacional del los Derechos Humanos. [Free and Informed Consultation and Prior Consent in light of International Human Rights Law]. Madrid.

Consejo Nacional Electoral. (2018). 2018, Resultados Referéndum y Consulta Popular. [2018, Referendum Results and Popular Consultation]. Obtenido de file:///C:/Users/USUARIO/Downloads/libro\%20resultados\%20referndum\%20y\%20 consulta\%20popular\%20de\%202018_parte_3.pdf

El Comercio. (30 de de agosto de 2018). La sentencia en contra de la minera Río Blanco fue ratificada. [The sentence against the Río Blanco mining company was ratified] Recuperado de https://n9.cl/2uo5n.

Erazo-Álvarez, J. C., \& Narváez-Zurita, C. I. (2020). Medición y gestión del capital intelectual en la industria del cuero - calzado en Ecuador. [Measurement and management of intellectual capital in the leather industry - footwear in Ecuador]. Revista Arbitrada Interdisciplinaria Koinonía, 437-467. doi:http://dx.doi.org/10.35381/r.k.v5i9.662

Fundación para el Debido Proceso. (2015). Derecho a la consulta y y al consentimiento previo, libre e informado en América Latina [Right to consultation and free, prior and informed consent in Latin America].Recuperado de: https://n9.cl/5pvq6. Washington, D.C. 
Grijalva Jiménez, A. (2009). "Introducción ¿Qué son los derechos olectivos?” en Los Derechos Colectivos. Hacia una efectiva comprensión y protección. ["Introduction What are the electoral rights?" in Collective Rights. Towards an effective understanding and protection]. Quito: Ministerio de Justicia y Derechos Humanos.

Instituto Interamericano de Derechos Humanos. (2016). El derecho a la Consulta Previa, Libre e Informada: Una mirada Crítica desde los pueblos Indigenas [The Right to Free, Prior and Informed Consultation: A Critical View from Indigenous Peoples].Recuperado de: https://n9.cl/sdc4. San José.

López Abad, J. (2016). La Consulta Libre, Previa e Informada en el Ecuador. [Free, Prior and Informed Consultation in Ecuador]. Quito: Centro de Derechos Económicos y Sociales-CDES.

Machuca Lozano, S. (2014). Análisis de la sentencia n. 001-10-SIN-CC.sobre acción de inconstitucionalidad en contra de la Ley de Minería. [Analysis of judgment No. 001 10-SIN-CC. On unconstitutional action against the Mining Law ], Recuperado de: https://n9.cl/dua0p. Revista de Derecho, n.ำ 21 UASB-Ecuador, 129-146.

Naciones Unidas Derechos Humanos Oficina del alto comisionado para los Derechos Humanos Colombia. (s.f.). El Derecho de los Pueblos Indígenasa la Consulta Previa, Libre e Informada [The Right of Indigenous Peoples to Free, Prior and Informed Consultation]. Recuperado de:https://n9.cl/eq18.

OIT. (1989). Convenio sobre pueblos indígenas y tribales. [Indigenous and Tribal Peoples Convention].

OIT. (2009). Los derechos de los pueblos indígenas y trubales en la práctica, una guia sobre el Convenio Número 169 de la OIT. [Indigenous and tribal peoples' rights in practice, a guide to ILO Convention No. 169 ]. Ginebra: Departamento de Normas Internacionales del Trabajo, 2009.

ONU. (13 de Septiembre de 2007). Declaración de Naciones Unidas Sobre los Derechos de los Pueblos Indígenas. [United Nations Declaration on the Rights of Indigenous Peoples]. Declaración de las Naciones Unidas sobre los derechos. Washinton, Estados Unidos : Resolución A/61/L.67 y Add.1.

Organización Internacional del Trabajo. (2016). Reporte regional: Colombia, Costa Rica, Guatemala, Chile. Lima: OIT, Oficina Regional para América Latina y el Caribe.[Regional report: Colombia, Costa Rica, Guatemala, Chile. Lima: ILO, Regional Office for Latin America and the Caribbean]. 112. 
Pesántez-Ochoa, J. A., Erazo-Álvarez, J. C., JVázquez-Calle, o. L., \& Narváez-Zurita, C. I. (2020). Vocales de las Juntas Provinciales Electorales: Principio de Independencia Electoral y Derecho a Participación Ciudadana [Members of the Provincial Electoral Boards: Principle of Electoral Independence and Right to Citizen Participation]. Iustitia Socialis, 182-207. doi:http://dx.doi.org/10.35381/racji.v5i1.608

Plan V. (14 de de mayo de 2018). 7 puntos para entender el conflicto minero en Río Blanco. [7 points to understand the mining conflict in Río Blanco] Recuperado de https://n9.cl/d1xnb.

Presidencia de la República del Ecuador. (13 de de junio de 2017). Presidecia del la República del Ecuador. [Presidency of the Republic of Ecuador]. Recuperado de https://n9.cl/wpzgd.

Reyes-Cueva, M. d., Erazo-Álvarez, J. C., Borja-Pozo, C. A., \& Narváez-Zurita, C. I. (2020). Mínima intervención penal en el juzgamiento contra delitos menores: Tutela judicial efectiva y reparación integral [Mínima intervención penal en el juzgamiento contra delitos menores: Tutela judicial efectiva y reparación integral]. Iustitia Socialis, 295-311. doi:http://dx.doi.org/10.35381/racji.v5i1.613

Salmoran, R. T. (2007). Teoría jurídica y "derecho comparado" una aproximación y un deslinde [Formal methodology of scientific research] Recuperado de: https://n9.cl/rg598. Universidad Nacional Autónoma de México, Facultad de Derecho, 29-49.

Tamayo y Tamayo, M. (2005). Metodología formal de la investigación científica [Formal methodology of scientific research]. Ciudad de Mexico: Editorial Limusa.

Vargas-Araujo, E. (2018). Una mirada crítica del derecho a la consulta previa, libre e informada. [A critical look at the right to free, prior and informed consultation]. Quito: Universidad Andina Simón Bolivar.

Vásquez-Ayerve, J. A., Narváez-Zurita, C. I., Pozo-Cabrera, E. E., \& Erazo-Álvarez, J. C. (2020). El alcance de la responsabilidad extracontractual del Estado por sentencia condenatoria reformada o revocada [The scope of the non-contractual responsibility of the State for a conviction amended or revoked]. Iustitia Socialis, 434-453. doi:http://dx.doi.org/10.35381/racji.v5i1.621 
Iustitia Socialis. Revista Arbitrada de Ciencias Jurídicas.

$$
\text { Año V. Vol. V. Nº. Julio - Diciembre } 2020
$$

Hecho el depósito de Ley: FA2016000064

ISSN: $2542-3371$

FUNDACIÓN KOINONIA (F.K). Santa Ana de Coro, Venezuela

David Sebastián Vázquez-Martínez; Juan Carlos Erazo-Álvarez; Cecilia Ivonne Narváez-Zurita; Enrique Eugenio Pozo-Cabrera

@2020 por los autores. Este artículo es de acceso abierto y distribuido según los términos y condiciones de la licencia Creative Commons Atribución-NoComercial-Compartirlgual 4.0 Internacional (CC BY-NC-SA 4.0) (https://creativecommons.org/licenses/by-nc-sa/4.0/). 